\title{
InGaAs/InAIAs SAGCMCT avalanche photodiode with high linearity and wide dynamic range
}

\author{
Yu Li (李 玉), Weifang Yuan (袁纬方), Ke Li (李 珂), Xiaofeng Duan (段晓峰)， Kai Liu (刘 凯), and \\ Yongqing Huang (黄永清) \\ State Key Laboratory of Information Photonics and Optical Communications, Beijing University of Posts and Telecommunications, Beijing 100876, China \\ *Corresponding author: xfduan@bupt.edu.cn \\ Received September 29, 2021 | Accepted November 10, 2021 | Posted Online December 2, 2021
}

Linearity is a very important parameter to measure the performance of avalanche photodiodes (APDs) under high input optical power. In this paper, the influence of the absorption layer on the linearity of APDs is carefully studied by using bandgap engineering with the structure model of separated absorption, grading, charge, multiplication, charge, and transit [SAGCMCT]. The simulated results show that in the hybrid absorption layer device structure the $1 \mathrm{~dB}$ compression point can be improved from $-9 \mathrm{dBm}$ to $-2.1 \mathrm{dBm}$ by increasing the proportion of the $\mathrm{p}$-type absorption layer. In the device structure with only one absorption layer, increasing the doping level of the absorption layer can also improve the $1 \mathrm{~dB}$ compression point from $-8.6 \mathrm{dBm}$ to $1.43 \mathrm{dBm}$ at a gain of 10 . Therefore, the absorption layer is very critical for the linearity of APDs.

Keywords: linearity; avalanche photodiode; dynamic range.

DOI: 10.3788/COL202220.022503

\section{Introduction}

Avalanche photodiodes (APDs) are widely used in optical communication systems. Compared with conventional photodiodes (PDs), APDs have a unique internal gain, which can achieve higher detection sensitivity ${ }^{[1-5]}$. Linearity is a very important performance of APDs for optical communication systems with operating rate greater than $10 \mathrm{Gbit} / \mathrm{s}$ or using a high-order modulation format, as well as an APD array ${ }^{[6-9]}$. For example, APDs used in the passive optical network (PON) need to maintain excellent linearity at high input power to deal with burst traffic in $\mathrm{PONs}^{[10,11]}$. Generally, compared with traditional PIN-PDs or uni-traveling carrier (UTC)-PDs, the linearity of APDs will deteriorate more seriously at higher optical input power ${ }^{[12-16]}$. It is considered that two factors lead to the poor linearity of APDs at high incident optical power. One reason is the thermal problem of APDs under high power operation. This is because APDs need to add a large reverse bias voltage to generate an internal gain, which also makes the thermal problem during operation more serious than that of ordinary PDs. Another reason is the space charge effect. The p-type field control layer is generally depleted at the working voltage. When holes with positive charge accumulate in the p-type field control layer, the negative charge in the $\mathrm{p}$-type field control layer will be offset. This will weaken the control effect of the p-type field control layer on the internal electric field distribution of the device. The electric field intensity of the multiplication layer (M-layer) will decrease, and the internal gain will also be reduced, resulting in the emergence of nonlinear effects. Nada et al. proposed an APD using a thin depleted absorber in $2015^{[17]}$. The fabricated APD showed improved linearity for input optical power over $-8 \mathrm{dBm}$. They proposed a novel APD structure in 2017, which has an absorber on both sides of the M-layer ${ }^{[6]}$. The fabricated APD exhibited an improved $1 \mathrm{~dB}$ compression point corresponding to an input power of $-6.3 \mathrm{dBm}$ at a gain of 10 . The advantage of this structure is that it can reduce the space charge effect, but both electrons and holes are injected into the M-layer, which will cause its noise to be greater than that of ordinary APDs. Jiang et al. studied the effect of the grading layer and charge layer on improving the linearity of APDs in $2019^{[18]}$. Through optimization, the $1 \mathrm{~dB}$ compression point of the device can be increased from $-10.44 \mathrm{dBm}$ to $-3.38 \mathrm{dBm}$. The bandwidth of $6.7 \mathrm{GHz}$ was obtained by this device.

To improve the linearity of APDs, it is necessary to solve the problem of hole accumulation under high input optical power. One of the effective ways to suppress the space charge effect is to use bandgap engineering. By changing the structure of the epitaxial layer and the doping level of each layer, the holes transport can be effectively accelerated, and the space charge effect can be alleviated. At present, there are two methods to alleviate the space charge effect by using bandgap engineering. Firstly, the bandgap difference between the M-layer and the absorption layer can be smoothed by adding a grading layer, which has been carefully studied by researchers ${ }^{[15]}$. Secondly, the energy band of the absorption layer can be changed by changing the structure 
and doping level of the absorption layer to accelerate transportation of holes. The influence of the absorption layer on the linearity of APDs has not been fully studied.

In this paper, the linearity of APDs is improved by using bandgap engineering. The influence of the absorption layer on APD linearity is carefully studied, and two separated absorption, grading, charge, multiplication, charge, and transit (SAGCMCT)-APD models with different absorption layer structures are established. The InAlAs M-layer thickness is $200 \mathrm{~nm}$. In the hybrid absorber device structure, increasing the proportion of the $\mathrm{p}$-type absorption layer can increase the $1 \mathrm{~dB}$ compression point from $-9 \mathrm{dBm}$ to $-2.1 \mathrm{dBm}$. In the device structure with only one absorption layer, the $1 \mathrm{~dB}$ compression point can be enhanced from $-8.6 \mathrm{dBm}$ to $1.43 \mathrm{dBm}$ by increasing the doping level of the absorption layer. Since increasing the doping level of the absorption layer will worsen the capacitance of the whole device, we adopted the SAGCMCT structure $^{[19]}$. The addition of the transit layer can reduce the capacitance of the device without affecting the transit bandwidth.

\section{Device Structure}

The epitaxial layer structure of the top-illuminated InGaAs/ InAlAs SAGCMCT-APD is shown in Fig. 1. It consists of a p-type contact layer, InGaAs absorption layer, InAlGaAs grading layer, p-type field control layer, $200 \mathrm{~nm}$ InAlAs M-layer, n-type field control layer, and transit layer. The total thickness of the whole absorber is $500 \mathrm{~nm}$. The diameter of the active region is $30 \mu \mathrm{m}$. The vertical central axis of the device is shown in A-A'. The mesa structure with a carefully designed diameter is used to limit the edge electric field of the M-layer. The p-type field control layer and n-type field control layer are used to adjust the electric field distribution of the whole device so that the M-layer maintains a high electric field. Since the transit velocity of holes is slower than that of electrons, adding a transit layer behind the n-type field control layer does not affect the transit bandwidth of the device, but it can increase the thickness of the depletion region and reduce the capacitance of the device.

A large number of electrons generated by the absorption layer will move to the M-layer and trigger an avalanche multiplication, causing the M-layer to produce more electron-hole pairs under high input optical power. The electrons generated in the M-layer will drift to the electrode and be collected, while

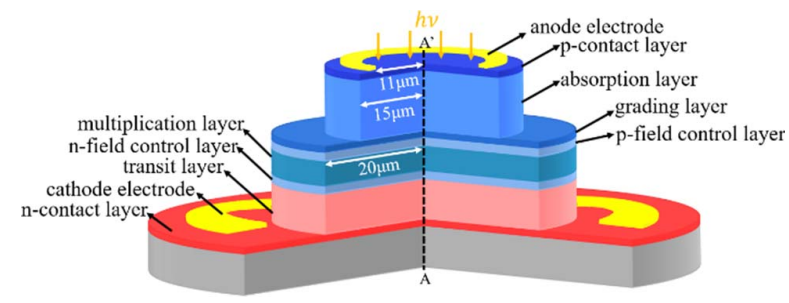

Fig. 1. Layer structure of the InGaAs/InAlAs SAGCMCT-APD. a large number of holes can also be collected by moving through the transit layer and absorption layer. Due to the slow movement velocity of holes, they tend to accumulate and lead to a space charge effect. Therefore, in order to alleviate the space charge effect, the transportation of holes needs to be accelerated. The effect of the absorbing layer on alleviating the space charge effect is worthy of careful study.

To study the influence of the absorption layer on the linearity of APDs, we designed two kinds of absorption layer structures. One is a hybrid absorption layer structure, and the other has only one absorption layer. The total thickness of the absorption layer is $500 \mathrm{~nm}$. Figures 2(a) and 2(b), respectively, show the band diagrams of the APD with these two structures under high input optical power. In the hybrid absorption layer structure, holes drift in the intrinsic absorption layer and relax directly in the p-type absorption layer. The effect of the p-type absorption layer thickness on the linearity of APDs can be explored. In the device structure with only one absorption layer, with the increase of the absorption layer doping level, the holes change from drift motion to direct relaxation, and the electrons change from drift motion to diffusion motion. The influence of the absorption layer doping level on the linearity of APDs can be explored.
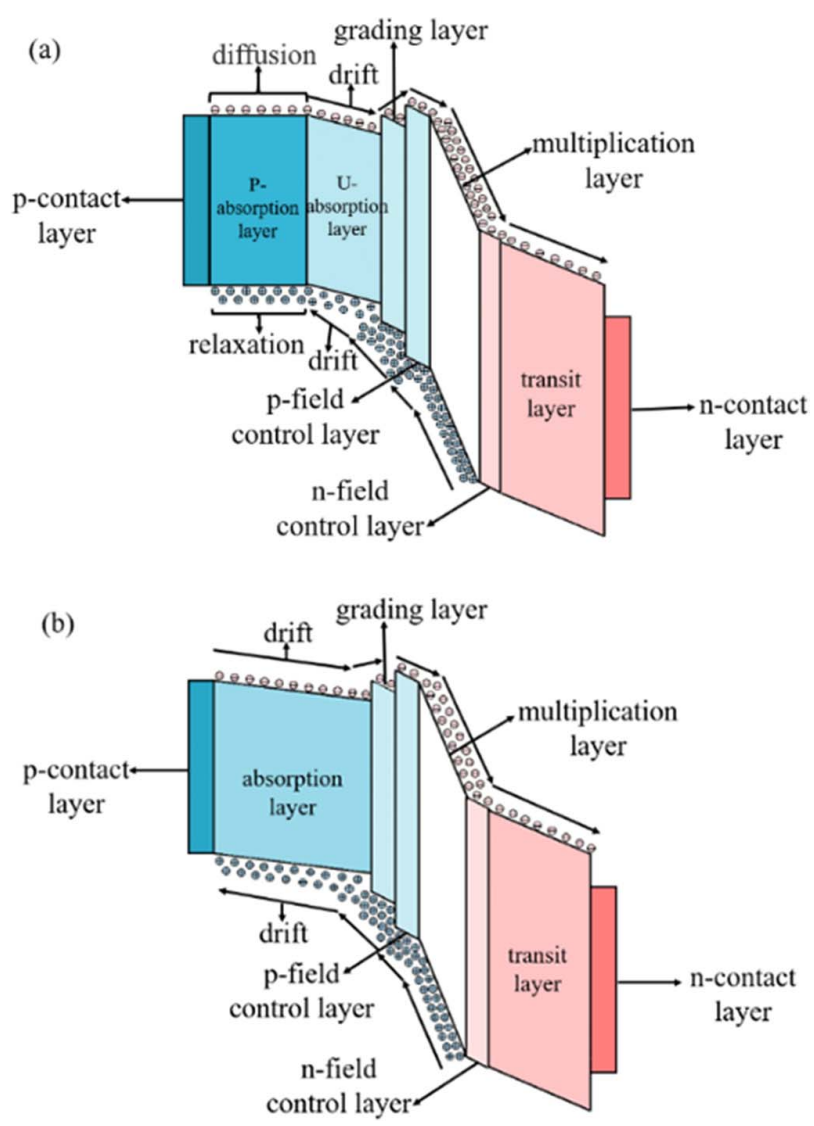

Fig. 2. (a) Band diagram of the hybrid absorption layer APD under high input optical power and (b) band diagram of the APD with only one absorption layer under high input optical power. 
Table 1. Material Parameters in the Simulation.

\begin{tabular}{|c|c|c|c|c|}
\hline Band-gap & eV & 0.75 & 0.99 & 1.44 \\
\hline Permittivity & $C^{2} /\left(N \cdot m^{2}\right)$ & 13.9 & 12.5 & 12.2 \\
\hline Selberherr's parameters $A_{n}, A_{p}$ & $\mathrm{~cm}^{-1}$ & $7 \times 10^{5}, 6.7 \times 10^{5}$ & $6.2 \times 10^{7}, 1 \times 10^{6}$ & $6.2 \times 10^{7}, 1 \times 10^{6}$ \\
\hline Selberherr's parameters $B_{n}, B_{p}$ & $\mathrm{~V} / \mathrm{cm}$ & $1.2 \times 10^{6}, 2 \times 10^{6}$ & $4 \times 10^{6}, 4 \times 10^{6}$ & $4 \times 10^{6}, 4 \times 10^{6}$ \\
\hline Selberherr's parameter $\beta$ & 1 & 1 & 1 & 1 \\
\hline Band-to-band parameter $A$ & $\mathrm{~V}^{-1} \cdot \mathrm{cm}^{-1} \cdot \mathrm{s}^{-1}$ & $1 \times 10^{19}$ & $1 \times 10^{19}$ & $5 \times 10^{19}$ \\
\hline
\end{tabular}

Results presented in this paper were obtained by the use of Silvaco technology computer aided design (TCAD). The Shockley-Read-Hall recombination model, Fermi-Dirac carrier statistics model, Auger recombination model, analytic concentration dependent mobility model, optical recombination model, Selberherr's impact ionization model, and band-to-band tunneling model are used in this simulation ${ }^{[20]}$. The material parameters of InGaAs, InAlGaAs, and InAlAs are listed in Table $1^{[21]}$.

Due to the space charge effect, the control effect of the field control layer on the electric field of APDs will be weakened under high input optical power. Thus, the electric field of the M-layer will be reduced, and the multiplication gain will also be decreased. Figures 3(a) and 3(b) show the simulated electric field distribution and the electron and hole concentration distribution in the vertical direction of the APD when the input optical power is $0.001 \mathrm{~mW}$ and $4.5 \mathrm{~mW}$, respectively. When the input optical power is $4.5 \mathrm{~mW}$, the electric field of the M-layer decreases by about $70 \mathrm{kV} / \mathrm{cm}$, and the electric field of the absorption layer increases. This is because the accumulation of holes leads to the weakening of the control effect of the p-type field control layer on the electric field. This is consistent with the above analysis.

\section{Simulation Results and Discussion}

In this section, we established an SAGCMCT-APD model with a hybrid absorption layer structure, and the total absorption layer thickness is $500 \mathrm{~nm}$. $R$ is used to represent the ratio of the p-type absorption layer thickness to the total absorption layer thickness. Figure 4(a) shows the simulated vertical direction (A-A') electric field distribution of the device under different $R$ values. The electric field of the M-layer is very uniform, and the electric field intensity is about $640 \mathrm{kV} / \mathrm{cm}$. The electric field range of the undoped absorption layer is between $88 \mathrm{kV} / \mathrm{cm}$ and $73 \mathrm{kV} / \mathrm{cm}$, while the electric field intensity of the $\mathrm{p}$-type absorption layer is about $0 \mathrm{kV} / \mathrm{cm}$. Due to the different thickness of the undoped absorption layer, the breakdown voltage $\left(V_{\mathrm{br}}\right)$ of the device is slightly different, but the gain of the device under $0.9 V_{\mathrm{br}}$ operation is the same at about 10. Figure 4(b) shows the energy band schematic diagram of the absorption layer. $W_{N}$ is used to represent the thickness of the non-depletion absorption layer, and $W_{D}$ is used to represent the thickness of the depletion absorption layer.

Figure 4(c) shows the absorption layer band diagram of the APD with a hybrid absorption layer structure at $0.9 V_{\text {br }}$ under different $R$ values. In the non-depleted absorption layer, the holes will relax directly, and, in the depleted absorption layer, the holes will drift. With the increase of non-depleted absorption layer thickness, the energy band will gradually be flattened, and the movement distance of holes will be shortened, which is beneficial to the transmission of holes. Figure 4(d) shows the simulated concentration distribution of the hole and electron in the vertical direction (A-A') at $R=0.2$ and $R=0.8$ under the input optical power of $0.5 \mathrm{~mW}$. At same input optical power, the hole concentration in the device at $R=0.2$ is larger than that at $R=0.8$. This indicates that compared with $R=0.8$, holes begin to accumulate when $R=0.2$.

Figure 5 shows the simulated input optical power dependence of linearity compression at the gain of 10 under different $R$ values. The $1 \mathrm{~dB}$ compression points of the device increase gradually with the increase of $R$. When the thickness of the p-type absorption layer is $100 \mathrm{~nm}$ and $400 \mathrm{~nm}$, the $1 \mathrm{~dB}$ compression points of the device are $-9 \mathrm{dBm}$ and $-2.1 \mathrm{dBm}$, respectively. Under high input optical power, holes will accumulate, resulting in the space charge effect. The linearity of the device will be reduced. The absorption layer is a long distance for holes to 
(a)

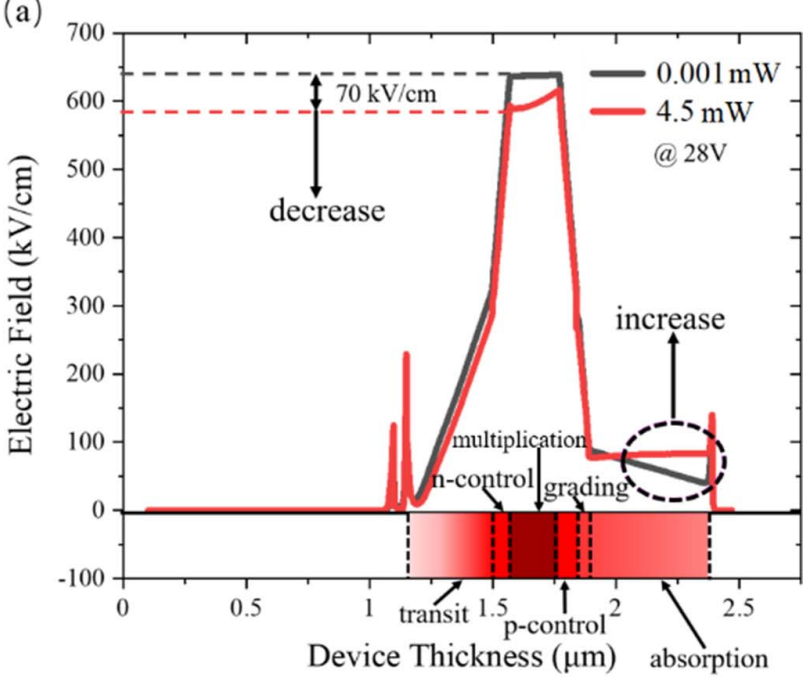

(b)

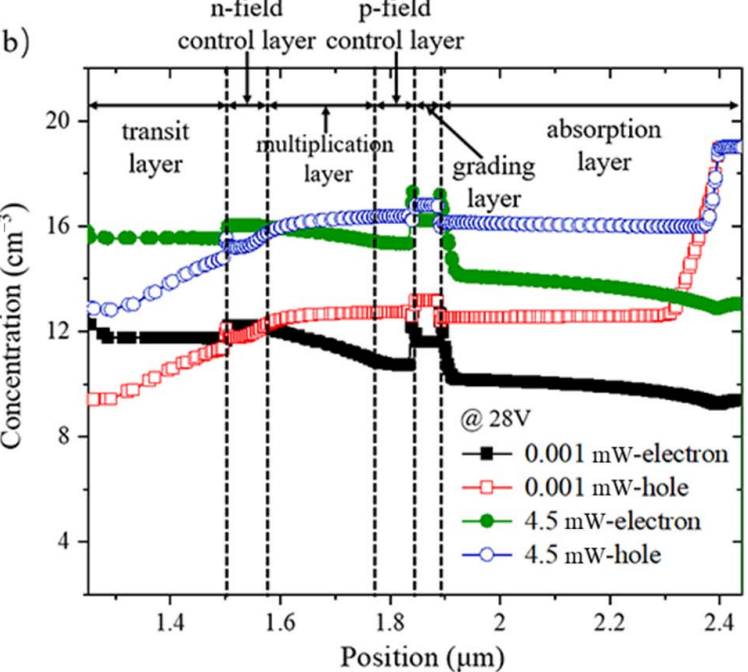

Fig. 3. Simulated vertical direction (A-A') (a) electric field distribution and (b) electrons and holes concentration distributions at the input optical power of $0.001 \mathrm{~mW}$ and $4.5 \mathrm{~mW}$.

transit. Increasing the thickness of the p-type absorption layer is equivalent to reducing the transmission distance of holes. The linearity of the device will be greatly improved.

In this section, we established an SAGCMCT-APD model with only one absorption layer, and the thickness of the absorption layer is $500 \mathrm{~nm}$. We set the doping level of the absorption layer to increase from $8 \times 10^{15}$ to $8 \times 10^{17} \mathrm{~cm}^{-3}$. Figure 6 (a) shows the simulated vertical direction (A-A') electric field distribution of the device under different absorption layer doping levels. The electric field intensity of the M-layer is about $610 \mathrm{kV} / \mathrm{cm}$. Figure 6(b) shows the absorption layer band diagram under different absorption layer doping levels. With the increase of the absorption layer doping level, the electric field of the absorption layer decreases gradually, and the energy band gradually becomes flat. The holes change from drift motion to relaxation, which greatly reduces the transit time of holes in the absorption layer. Figure 6(c) shows the simulated concentration distribution of the hole and electron in the vertical direction (a)

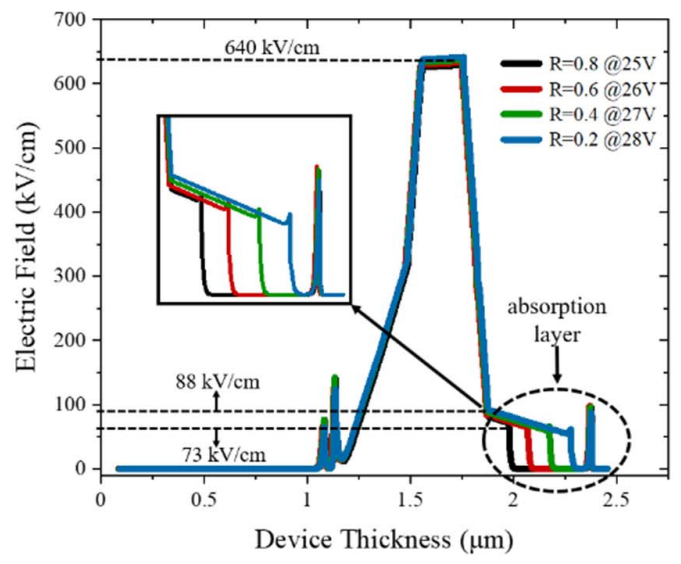

(b)
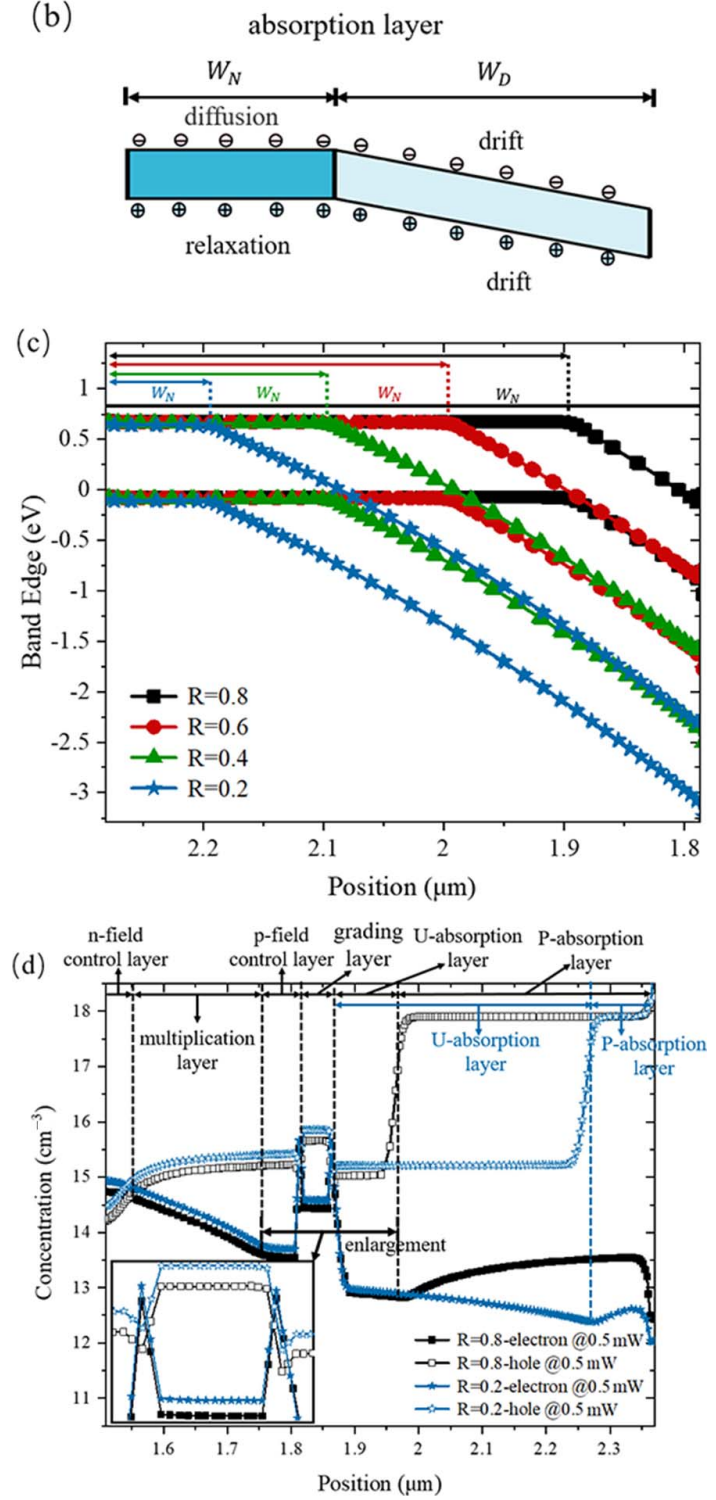

Fig. 4. (a) Simulated vertical direction (A-A') electric field distribution of the device, (b) energy band schematic diagram of the absorption layer, (c) absorption layer band diagram at $0.9 V_{\mathrm{br}}$ under different $R$ values, and (d) simulated hole and electron concentration distribution in the vertical direction $\left(A-A^{\prime}\right)$ at $R=0.2$ and $R=0.8$ under the input optical power of $0.5 \mathrm{~mW}$. 


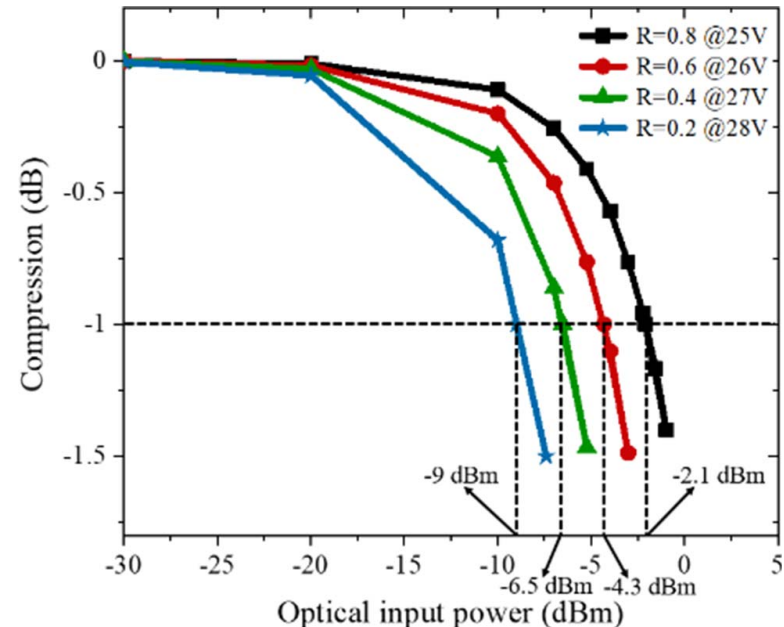

Fig. 5. Simulated input optical power dependence of linearity compression at the gain of 10 under different $R$ values.

(A-A') of the device at the input optical power of $1 \mathrm{~mW}$ under different absorption layer doping levels. When the absorption layer doping level is $8 \times 10^{15} \mathrm{~cm}^{-3}$, the hole concentration is significantly higher than that when the absorption layer doping level is $8 \times 10^{17} \mathrm{~cm}^{-3}$ under the same input optical power. When the incident light power is $1 \mathrm{~mW}$, the device with the absorption layer doping level of $8 \times 10^{15} \mathrm{~cm}^{-3}$ has serious hole accumulation, which will produce a space charge effect and greatly affect the linearity of the device.

Figure 7 shows the simulated optical input power dependence of linearity compression at the gain of 10 under different absorption layer doping levels. With the increase of the doping level of the absorption layer, the $1 \mathrm{~dB}$ compression point of the device increases from $-8.6 \mathrm{dBm}$ to $1.43 \mathrm{dBm}$. These results indicate that the rapid transport of holes in the absorption layer plays an important role in reducing the space charge effect and improving the linearity of the device at high input optical power.

From Fig. 8(a), when the input optical power is $1.5 \mathrm{~mW}$, the photocurrent of the device increases with the increasing doping levels of the absorption layer. The gain of the device can also be improved. Figure 8(b) shows the simulation bandwidth of the device at the gain of 10 under different absorption layer doping levels. The $3 \mathrm{~dB}$ bandwidth of the device is about $8 \mathrm{GHz}$ to $8.5 \mathrm{GHz}$. Compared with Ref. [15], the $1 \mathrm{~dB}$ compression point is $-3.38 \mathrm{dBm}$, and the bandwidth is $6.7 \mathrm{GHz}$. Our optimized device obtains a bandwidth of $8 \mathrm{GHz}$ and a $1 \mathrm{~dB}$ compression point of $1.43 \mathrm{dBm}$. The final optimized structure parameters of the SAGCMCT-APD are shown in Table 2.

\section{Conclusions}

In summary, the space charge effect can be alleviated, and the linearity of APDs can be improved by using bandgap engineering. The absorption layer is also crucial for improving the linearity of APDs because the holes need to be transmitted in a thicker absorption layer. In the hybrid absorption layer structure,
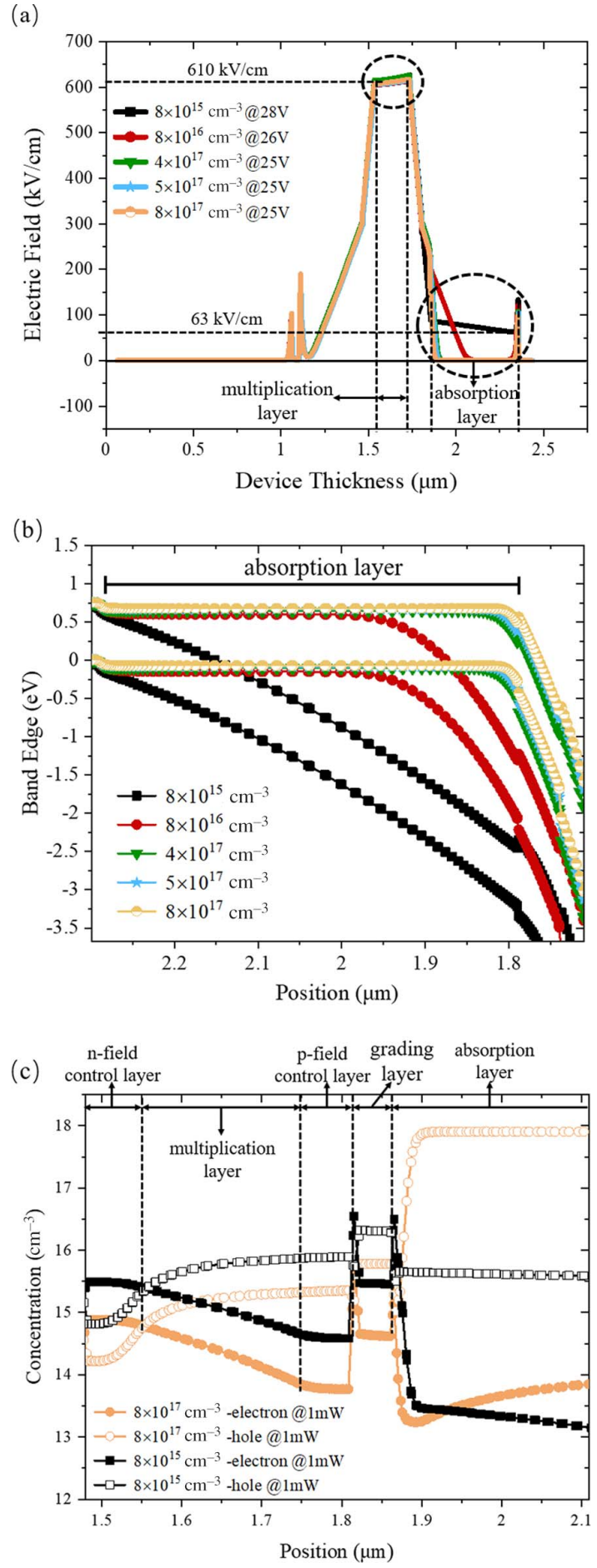

Fig. 6. Simulated vertical direction (A-A') (a) electric field distribution and (b) absorption layer band diagram of the device under different absorption layer doping levels; (c) simulated hole and electron concentration distribution in the vertical direction (A-A') of the device at the gain of 10 under different absorption layer doping levels.

increasing the ratio of the p-type absorption layer thickness to the total absorption layer thickness can shorten the transit time of holes and improve the linearity of APDs. In the single absorption layer structure, increasing the doping level of the 


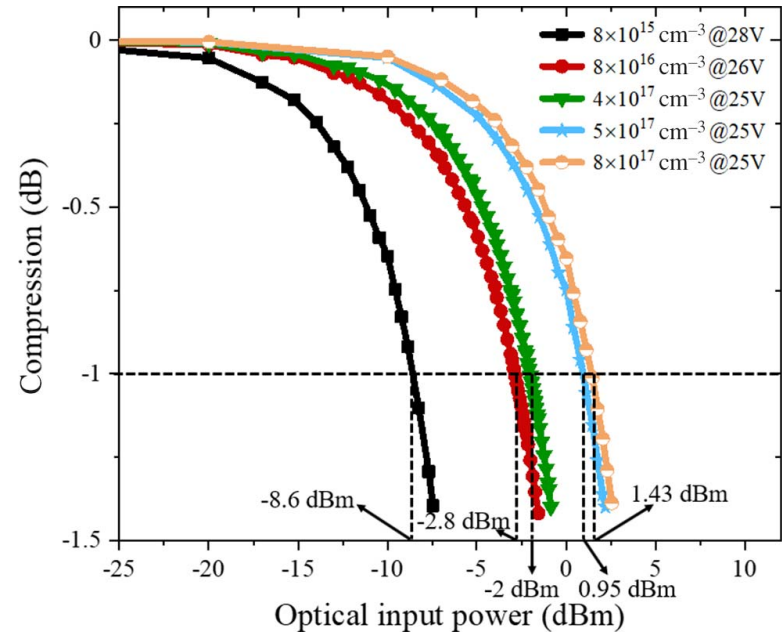

Fig. 7. Simulated optical input power dependence of linearity compression at the gain of 10 under different absorption layer doping levels.
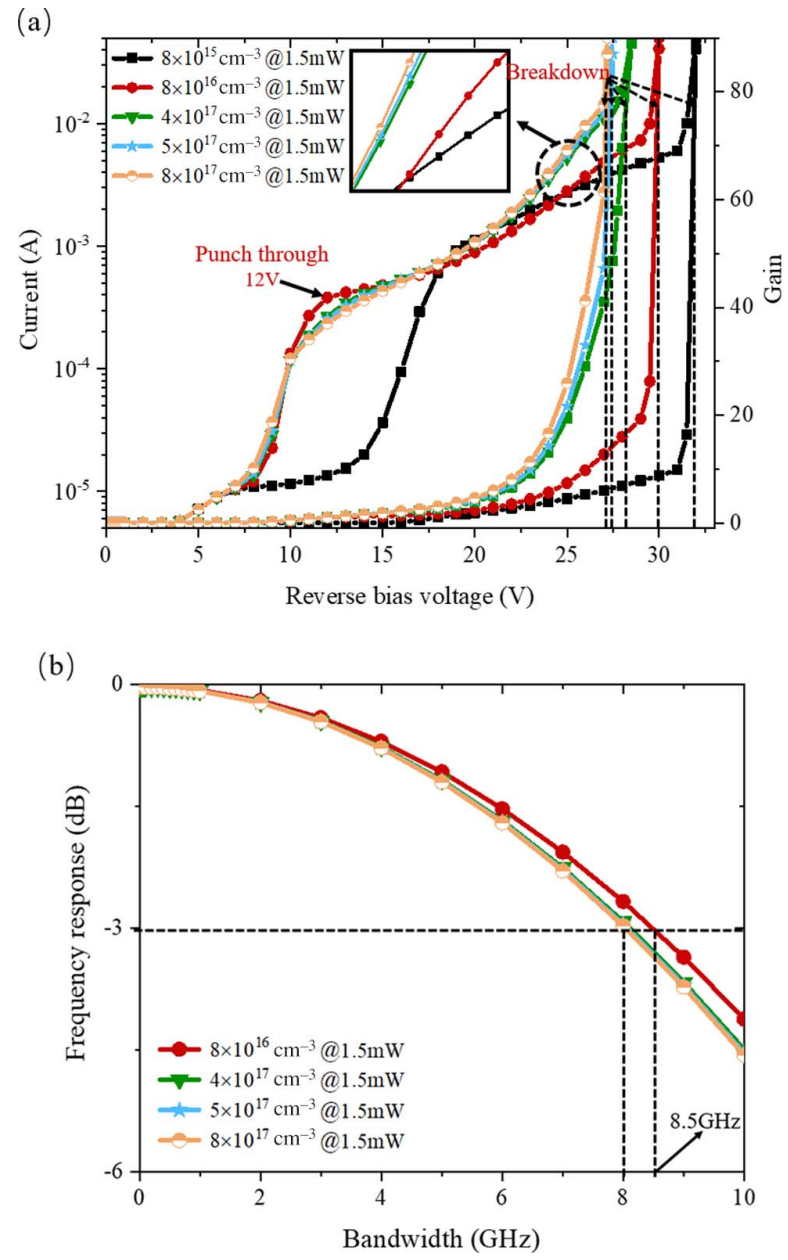

Fig. 8. (a) Simulated photocurrent and gain of the device under different absorption layer doping levels and (b) simulated bandwidth of the device at the gain of 10 under different absorption layer doping levels. Compared with Ref. [15], the $1 \mathrm{~dB}$ compression point is $-3.38 \mathrm{dBm}$, and the bandwidth is $6.7 \mathrm{GHz}$. Our optimized device obtains a bandwidth of $8 \mathrm{GHz}$.
Table 2. Structure Parameters of the SAGCMCT-APD.

\begin{tabular}{lcc}
\hline Layer & Thickness $(\mathrm{nm})$ & Doping $\left(\mathrm{cm}^{-3}\right)$ \\
\hline P-InGaAs contact layer & 50 & $1 \times 10^{19}$ \\
InGaAs absorption layer & 500 & $8 \times 10^{17}$ \\
InAlGaAs grading layer & 50 & $3 \times 10^{17}$ \\
P-InAlAs field control layer & 70 & $3 \times 10^{17}$ \\
InAIAs M-layer & 200 & $1 \times 10^{15}$ \\
N-InAlAs field control layer & 70 & $3 \times 10^{17}$ \\
InP-transit layer & 350 & $7 \times 10^{16}$ \\
N-InGaAs contact layer & 50 & $1 \times 10^{19}$ \\
\hline
\end{tabular}

absorption layer can also make the holes shift from drift motion to relaxation and improve the linearity of the device. In the APD designed with only one absorption layer, the $1 \mathrm{~dB}$ compression point can reach $1.43 \mathrm{dBm}$ when the doping level is set to $8 \times 10^{17} \mathrm{~cm}^{-3}$. The device achieves $8 \mathrm{GHz}$ bandwidth at a gain of 10. The improvement principle is similar to UTC-PD with high linearity.

\section{Acknowledgement}

This work was supported by the National Key Research and Development Program of China (No. 2018YFB2200803).

\section{References}

1. L. E. Tarof, J. Yu, R. Bruce, D. G. Knight, T. Baird, and B. Oosterbrink, "Highfrequency performance of separate absorption grating, charge, and multiplication InP/InGaAs avalanche photodiodes," IEEE Photon. Technol. Lett. 5, 672 (1993).

2. C. Liao, J. Wang, H. Lu, X. Peng, J. Guo, R. Li, Z. Wei, J. Zhou, J. Feng, and S. Liu, "Experimental characterization improving the design of InGaAs/InP APD for single photon detection," Chin. Opt. Lett. 3, S31 (2005).

3. P. Zhou, C. Liao, Z. Wei, C. Li, and S. Yuan, "Determination of breakdown voltage of $\operatorname{In}_{0.53} \mathrm{Ga}_{0.47} \mathrm{As} / \mathrm{InP}$ single photon avalanche diodes," Chin. Opt. Lett. 9, 010402 (2011).

4. J. C. Campbell, S. Demiguel, F. Ma, A. Beck, X. Zheng, X. Li, J. D. Beck, M. A. Kinch, A. Huntington, L. A. Coldren, J. Decobert, and N. Tscherptner, "Recent advances in avalanche photodiodes," IEEE J. Sel. Top. Quantum Electron. 10, 777 (2004).

5. J. C. Campbell, "Recent advances in avalanche photodiodes," J. Lightwave Technol. 34, 278 (2016).

6. M. Nada, Y. Yamada, and H. Matsuzaki, "A high-linearity avalanche photodiodes with a dual-carrier injection structure," IEEE Photonics Technol. Lett. 29, 1828 (2017).

7. M. Nada, T. Hoshi, H. Yamazaki, T. Hashimoto, and H. Matsuzaki, "Linearity improvement of high-speed avalanche photodiodes using thin depleted absorber operating with higher order modulation format," Opt. Express. 23, 27715 (2015).

8. M. Nada, T. Yoshimatsu, Y. Muramoto, H. Yokoyama, and H. Matsuzaki, "Design and performance of high-speed avalanche photodiodes for 100Gb/s systems and beyond," J. Lightwave Technol. 33, 984 (2015). 
9. L. Zhang, Y. Yang, W. Xia, X. Zhu, W. Chen, and Y. Lu, "Linearity of quadrant avalanche photodiode in laser tracking system," Chin. Opt. Lett. 7, 728 (2009).

10. T. Tsutsumi, T. Sakamoto, Y. Sakai, T. Fujiwara, H. Ou, Y. Kimura, and K. Suzuki, "Long-reach and high-splitting-ratio 10G-EPON system with semiconductor optical amplifier and N:1 OSU protection," J. Lightwave Technol. 33, 1660 (2015).

11. M. Nada, M. C. Chen, H. Katsurai, Y. Nakanishi, and K. Sano, "Practically implementable high-sensitivity $10-\mathrm{Gbit} / \mathrm{s}$ avalanche photodiode using inverted p-down design,” IEICE Electron Expr. 18, 20210142 (2021).

12. A. Beling, H. Pan, H. Chen, and J. C. Campbell, "Measurement and modeling of a high-linearity modified unitraveling carrier photodiode," IEEE Photon. Technol. Lett. 20, 1219 (2008).

13. W. Sun, Y. Fu, Z. Lu, and J. C. Campbell, "Study of bandwidth enhancement and non-linear behavior in avalanche photodiodes under high power condition," J. Appl. Phys. 113, 044509 (2013).

14. C. Wu, J. Zhang, X. Peng, and W. Qian, "Improving the detection range of APD detection system by waveform integration," Infrared Laser Eng. 49, 20200149 (2020).
15. C. Shen, L. Yang, H. Guo, D. Yang, L. Chen, and L. He, "Numerical simulation of high-operating-temperature MWIR HgCdTe APD detectors," J. Infrared Millim. Waves 40, 576 (2021).

16. D. Zheng, X. Su, Y. Xu, and Z. Niu, "High gain and low dark current AlInAsSb avalanche photodiodes grown by quaternary digital alloys," J. Infrared Millim. Waves 40, 172 (2021).

17. M. Nada, T. Hoshi, H. Yamazaki, T. Hashimoto, and H. Matsuzaki, "Linearity improvement of high-speed avalanche photodiodes using thin depleted absorber operating with higher order modulation format," Opt. Express 23, 27715 (2015).

18. Y. Jiang and J. Chen, "Optimization of the Linearity of InGaAs/InAlAs SAGCM APDs,” J. Lightwave Technol. 37, 3459 (2019).

19. H. Wang, X. Yang, R. Wang, T. He, and K. Liu, "Low dark current and high gain-bandwidth product of avalanche photodiodes: optimization and realization," Opt. Express 28, 16211 (2020).

20. Silvaco, ATLAS User's Manual Device Simulation Software (Silvaco, 2010).

21. O. S. Abdulwahid, J. Sexton, I. Kostakis, K. Ian, and M. Missous, "Physical modelling and experimental characterisation of InAlAs/InGaAs avalanche photodiode for $10 \mathrm{~Gb} / \mathrm{s}$ data rates and higher," IET Optoelectron. 12, 5 (2018). 